\title{
Physical Activity and Long-Term Mortality Risk in Older Adults with and without Cardiovascular Disease: A Nationwide Cohort Study
}

\author{
Or Shaked ${ }^{\mathrm{a}}$ Gali Cohen $^{\mathrm{a}} \quad$ Abigail Goshen $^{\mathrm{a}}$ Tal Shimony $^{\mathrm{b}}$ Tamar Shohat $^{\mathrm{a}} \mathrm{b}$ \\ Yariv Gerber ${ }^{a}$ \\ aDepartment of Epidemiology and Preventive Medicine, School of Public Health, Sackler Faculty of Medicine, Tel \\ Aviv University, Tel Aviv, Israel; ' Israel Center for Disease Control, Israel Ministry of Health, Ramat Gan, Israel
}

\section{Keywords}

Physical activity · Mortality · Older adults · Cardiovascular disease $\cdot$ Epidemiology $\cdot$ MABAT

\begin{abstract}
Aims: To evaluate the association between physical activity (PA) levels and mortality among older adults, to determine whether it differs according to cardiovascular disease (CVD) status, and to assess the optimal weekly duration of PA associated with subsequent survival. Methods: Participants $(n=1,799)$ were drawn from a national survey conducted from 2005 to 2006 , constituting Israeli adults aged $\geq 65$ years. Sociodemographic, clinical, behavioral, and psychosocial data were collected via interview at study entry. Based on a detailed PA questionnaire and according to published guidelines, participants were classified as sufficiently active, insufficiently active, and inactive. CVD status was self-reported. Mortality data (last follow-up, December 2016) were obtained from the Israeli Ministry of Health. Using Cox models, inverse probability weighted hazard ratios (HRs) for mortality, based on propensity score, were estimated for PA categories. Results: Among the participants at baseline (mean age, 74.6 years), 559 (31.1\%) were sufficiently active, 506
\end{abstract}

karger@karger.com www.karger.com/ger

Karger"

GOPEN ACCESS
C 2021 The Author(s)

Published by S. Karger AG, Basel

This is an Open Access article licensed under the Creative Commons Attribution-NonCommercial-4.0 International License (CC BY-NC) (http://www.karger.com/Services/OpenAccessLicense), applicable to the online version of the article only. Usage and distribution for commercial purposes requires written permission.
(28.1\%) were insufficiently active, and 734 (40.8\%) were inactive. During follow-up (mean, 9.0 years), 684 participants (38.0\%) died. PA was inversely associated with mortality, with propensity score-adjusted HRs ( $95 \%$ confidence intervals) of $0.84(0.71-1.01)$ in insufficiently and $0.73(0.61-0.88)$ in sufficiently active participants ( $p_{\text {trend }}<0.001$ ). No PA-byCVD interaction was detected on multiplicative scale ( $p=$ $0.36)$ or additive scale $(p=0.58)$. A monotonic survival benefit was observed until 150 min of PA per week, beyond which no further gain was apparent. Conclusions: In a nationwide cohort of older adults, nearly $70 \%$ did not meet the guideline for PA. PA engagement was inversely associated with long-term mortality risk, similarly in individuals with and without CVD. A maximum survival advantage was achieved at around 150 min of exercise per week.

(c) 2021 The Author(s)

Published by S. Karger AG, Basel

\section{Introduction}

Over the years, observational studies have consistently demonstrated a strong, dose-response inverse association between physical activity (PA) and mortality across different countries and populations, including older adults
Correspondence to:

Yariv Gerber, yarivg@tauex.tau.ac.il 
(aged $\geq 65$ years) [1]. The association was also shown among patients with cardiovascular disease (CVD) $[2,3]$. The older adult population is characterized by a high prevalence of chronic illnesses, functional disabilities, cognitive decline, and decreased psychological functioning [4]. These characteristics correlate both with one's likelihood of being physically active and his/her survival [5-8]. However, these parameters were not adequately taken into consideration in many of the previous studies examining the association between PA and survival in the elderly [9].

PA can improve CVD risk factor profiles, prevents primary and secondary cardiovascular events in both young and older adults [10]. Cardiovascular pathophysiology and physiology of aging share common biological pathways, such as insulin resistance and inflammation [11], which may result in alterations in the cardiovascular system as well as in muscles, bones, and nerves, all of which tend to erode exercise capacity and to increase susceptibility to CVD [12]. Taken together, CVD status may modify the potential benefits of PA, marking the importance of quantifying the association between PA and survival in older adults with and without CVD. To date, only a few studies have addressed this issue. Bembom et al. [13], studying a cohort of older adults from Sonoma, CA (median age, 70 years), showed that the mortality-sparing effect of PA is independent of the presence or absence of underlying CVD. In contrast, Jeong et al. [14], studying a large population-based cohort from the Republic of Korea (median age, 59.5 years), concluded that individuals with CVD may benefit from PA to a greater extent than do healthy subjects without CVD. Notably, both studies used a relatively short follow-up duration of up to 5 years which makes them particularly prone to "healthy exerciser bias," a special type of selection bias where healthy and relatively fit elderly individuals are more likely to participate in vigorous PA [9]. Hence, further investigation in this field is warranted. In addition, as PA was more strongly associated with survival in elderly than younger subjects [1], the optimal weekly duration of PA among the elderly is yet to be clear [15].

We utilized an extensive dataset of 1,799 Israeli older adults aged $\geq 65$ years followed-up over nearly a decade to examine the long-term association between PA and allcause mortality in a nationwide cohort, while appropriately accounting for a wide range of potential confounders and assessing the modifying role of CVD on the association. Further, we attempted to identify the cumulative weekly duration of PA associated with subsequent survival in this population.

\section{Materials and Methods}

\section{Study Design and Setting}

The cohort comprised all participants interviewed in the first National Health and Nutrition Survey of the Elderly in Israel ("Mabat Zahav"), carried out between 2005 and 2006 by the Israel Center for Disease Control and the Nutrition Department of the Israel Ministry of Health [16]. The survey population constituted a random sample of Israeli citizens aged 65 years or older, with the sampling framework provided by the 2 major health maintenance organizations in Israel (Clalit Health Services and Maccabi Health Services), representing approximately $86 \%$ of all elderly individuals in Israel. The final sample included 1,852 community-dwelling participants residing in Israel for at least 1 -year. Data were obtained via a personal interview in the interviewee's place of residence (own home or retirement home) using a structured questionnaire. Among the participants interviewed, 53 were excluded because of severe cognitive impairment $(n=46)$ or incomplete questionnaires $(n=7)$, yielding 1,799 participants (for whom survey data are available online at the Ministry of Health government website [17]).

\section{Assessment of PA}

Leisure-time PA was self-reported during the baseline interview, based on a standard questionnaire (for full questionnaire see Ministry of Health website - English version available [18]), as previously described in detail [19]. In 2 sets of questions, participants were asked about their PA habits. One set referred to vigorous-intensity activity and another set addressed any type of moderate PA that lasted at least $10 \mathrm{~min}$. Participants reported the frequency (times per week) and average time they devoted to each specific activity, as follows: walking outdoors or on a treadmill, jogging, swimming, bike riding or stationary cycling, light exercise (such as yoga, the Feldenkrais Method, the Alexander technique, and light gymnastics), body shaping, and strength training; an "other activity" option was also offered. Based on the reported total weekly time of PA and intensity, participants were classified into 3 PA categories according to the official American College of Sports Medicine (ACSM) guideline [20]: sufficiently active, insufficiently active, or inactive. Individuals who performed moderate PA for at least 150 min per week or a vigorous-intensity activity for at least 75 min per week or a combination of the 2 were classified as sufficiently active; those who engaged in PA but in a lesser amount than these definitions were classified as insufficiently active; and those who reported no activity or activity less than once a week were classified as inactive.

\section{Cardiovascular Status Assessment}

Cardiovascular morbidity was self-reported during the baseline interview. Participants were classified as having CVD if they answered "Yes" to one of the following questions: "Did a doctor tell you in the past that you have: myocardial infarction/heart failure/other heart disease?" or reported having undergone coronary artery bypass grafting or percutaneous coronary intervention.

\section{Mortality Assessment}

Data on all-cause mortality (from study entry throughout December 31,2016$)$ were obtained by linking the cohort (via national identification numbers) to the nationwide database of death, managed by the Ministry of Health. 
Table 1. Selected characteristics of study cohort by physical activity categories before and after inverse probability of treatment weighting ${ }^{\mathrm{a}}$

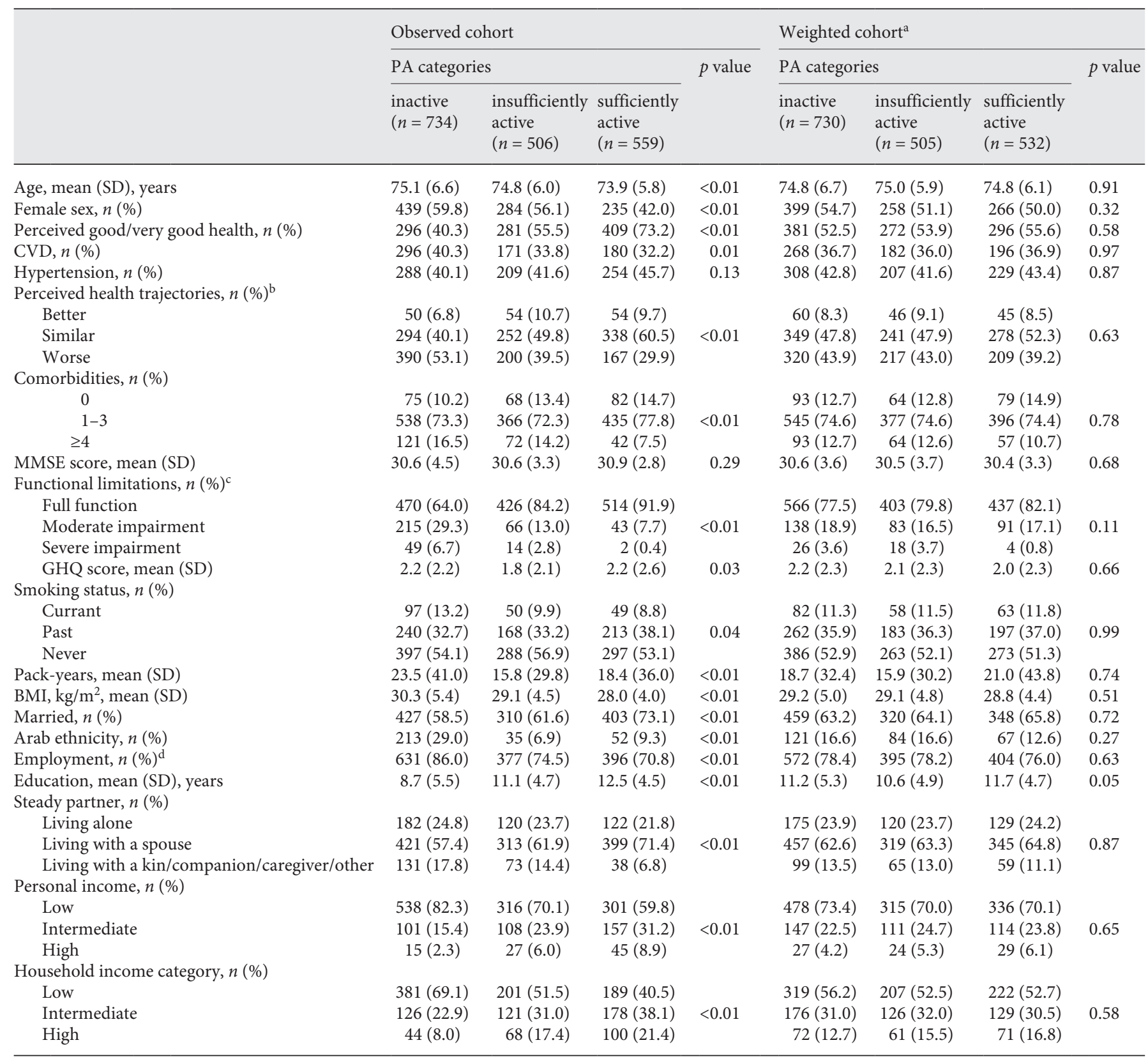

ADL, activities of daily living; BMI, body mass index; GHQ, General Health Questionnaire; MMSE, Mini-Mental State Examination; SD, standard deviation; CVD, cardiovascular disease. ${ }^{\mathrm{a}}$ Weighted by inverse probability of treatment, as described in the Statistical Analyses section. ${ }^{\mathrm{b}}$ Perceived health at the time of the interview in comparison to preceding year. ${ }^{\mathrm{c}}$ Evaluated via ADL score, as described in the Statistical Analyses section. ${ }^{\mathrm{d}}$ Including salaried/ unsalaried/volunteer.

\section{Additional Covariates}

Comprehensive information on sociodemographic variables, health conditions, disabilities, mental health, cognitive function, anthropometric measures, and nutritional status was obtained at the baseline interview [16]. Baseline covariates included age, sex, multimorbidity index, smoking status and pack-years, current perceived health, perceived health compared to the year preceding the interview, CVD, hypertension, functional status, mental health, cognitive status, body mass index, ethnicity, marital status, living with a steady partner, employment, education, personal income, and household income. Full description of baseline covariates is available in online suppl. material; see www.karger.com/doi/10.1159/000518169 for all online suppl. material. 


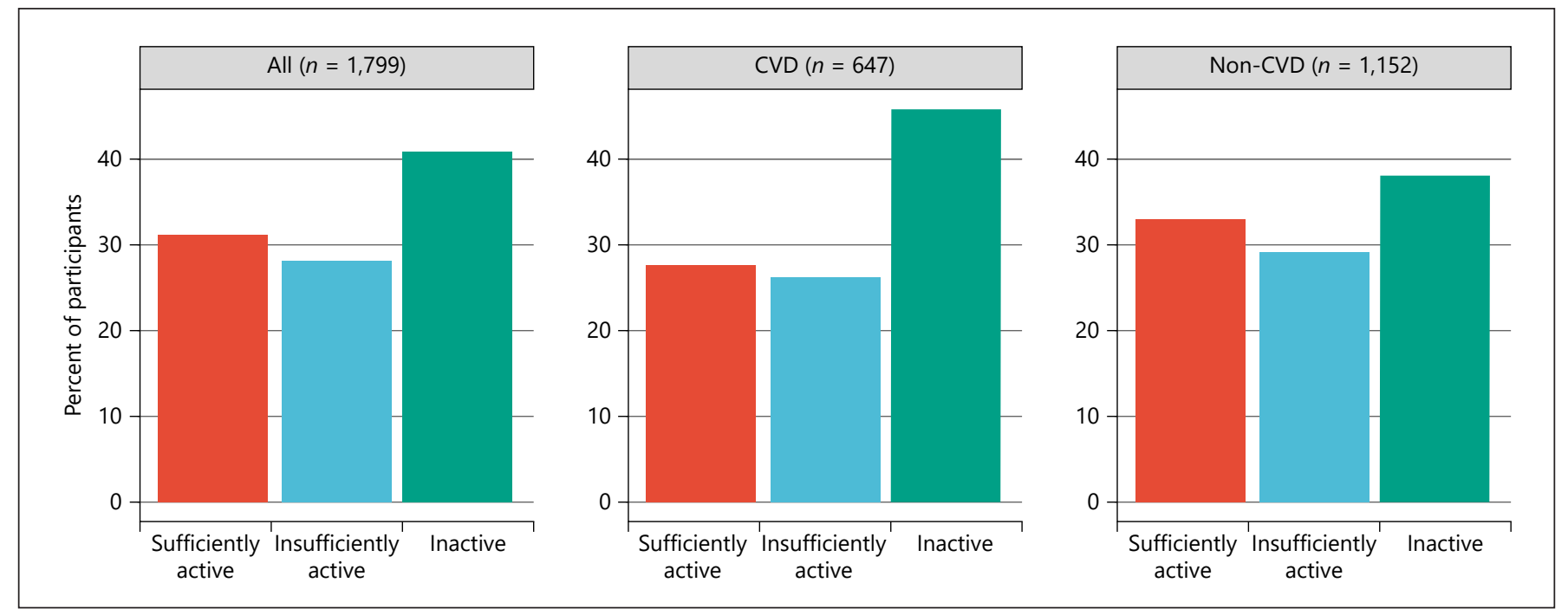

Fig. 1. Distribution of PA categories in the entire cohort and by CVD status. $p=0.01$ for the comparison of distributions between CVD and non-CVD individuals. CVD, cardiovascular disease, PA, physical activity.

\section{Statistical Analysis}

A propensity score was constructed using a multinomial logistic regression, through which the probability of being classified into a specific PA category (inactive, insufficiently active, or sufficiently active), conditional on measured baseline covariates, was estimated. Inverse probability weights were calculated using the propensity score [21], by weighting each participant in each PA category inverse to her/his probability of being classified into that specific PA category. In this manner, we created a pseudopopulation in which the distribution of measured baseline covariates is independent of PA category; thus, accounting for differences between the individuals in PA categories that could influence the outcome. Because of the instability that can be induced by extreme weights, stabilized weights were used to preserve the original sample size [22]. Truncation was additionally applied by resetting observations with weights below the 1st percentile and above the 99th percentile to the values of the 1st and 99th percentiles, respectively $[21,23]$. Mortality rates in PA categories, stratified by CVD status, were estimated according to inverse probability weights based on the propensity score. Subsequently, weighted Cox proportional hazards regression models with age as the time scale were constructed to estimate the hazard ratios (HRs) and 95\% confidence intervals (CIs) for all-cause mortality in PA categories. HRs were further estimated for each PA category stratified by CVD status. Linear trend across PA categories was tested by treating PA categories as a continuous variable in the regression model. We repeated the main analysis by using a conventional Cox model adjusted for selected baseline covariates. Effect modification of baseline CVD on the PA-mortality association was examined by testing a twoway interaction term of PA-by-CVD in a regression model which included PA and CVD status as the main effects. Additive interaction was tested by comparing adjusted risk differences as estimated through stratified proportional hazards models. In addition, we calculated the total time (minutes) of PA summing all the activities reported. We then applied spline methodology using a penalized spline term to assess the relationship between total time of PA and mortality in a Cox regression model. The proportional hazards assumption was tested using the Schoenfeld residuals, with no violations found in any of the models. Analyses were performed using R software, version 3.4.1 (R Development Core Team).

\section{Results}

At study entry, the mean (standard deviation) age of the 1,799 participants was $74.6(6.2)$ years, and 958 (53.3\%) were women. Overall, 817 participants (45.4\%) were current or past smokers, $813(45.2 \%)$ perceived their health as poor, and $389(21.6 \%)$ had some level of functional impairment. At baseline, 734 participants (40.8\%) did not engage in any type of PA, $506(28.1 \%)$ engaged in PA below the ACSM guideline, and 559 (31.1\%) met the guideline. Baseline characteristics distributed differently across PA categories. Compared with sufficiently active participants, inactive participants were slightly older and more likely to be female, with higher body mass index, current smokers and of lower socioeconomic status (SES) as measured by years of education, personal income, and household income. Sufficiently active participants reported better general health, with lower CVD prevalence than insufficiently active and inactive participants. Weighting using the inverse propensity score resulted in balanced PA groups with regard to baseline characteristics (Table 1).

At baseline, 647 (36.0\%) participants reported a history of CVD. Among the latter group, 180 (27.9\%) were 
Fig. 2. Adjusted mortality rate across PA categories among individuals with and without CVD. Rates are weighted according to inverse of propensity score for PA category. CVD, cardiovascular disease; PA, physical activity.

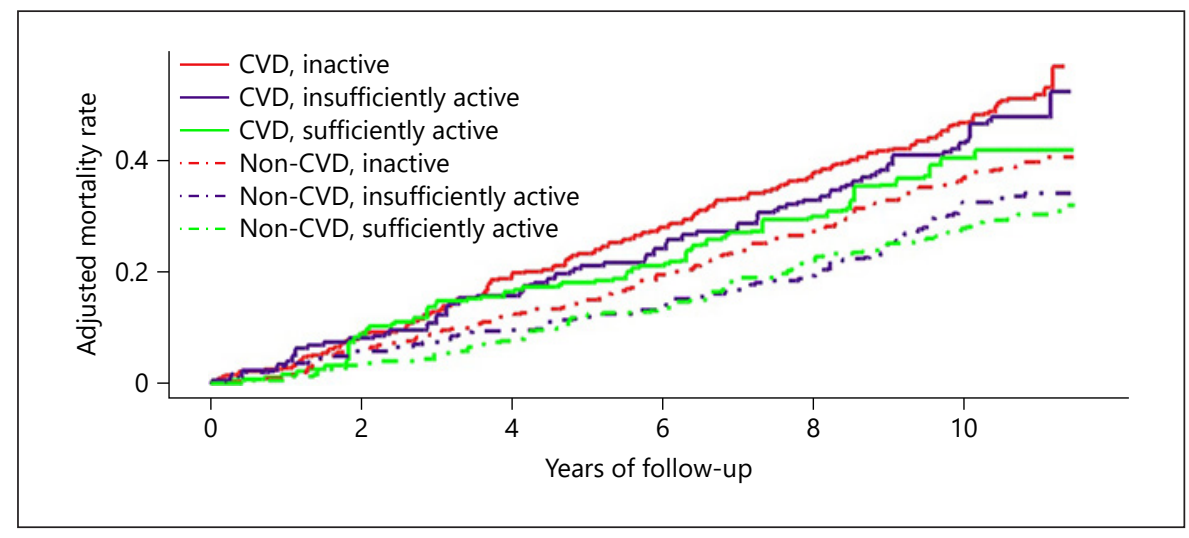

Table 2. HRs and 95\% CIs for mortality associated with physical activity category

\begin{tabular}{|c|c|c|c|c|}
\hline $\mathrm{PA}$ & $\begin{array}{l}\text { Number of } \\
\text { subjects }\end{array}$ & Unadjusted & $\begin{array}{l}\text { Multivariable } \\
\text { adjustment }^{\mathrm{a}}\end{array}$ & $\begin{array}{l}\text { Inverse probabil- } \\
\text { ity weighting }\end{array}$ \\
\hline \multicolumn{5}{|c|}{ All cohort $(n=1,799 ;$ number of cases $=684)$} \\
\hline Inactive & 734 & 1 & 1 & 1 \\
\hline Insufficiently active & 506 & $0.64(0.54,0.77)$ & $0.80(0.66,0.97)$ & $0.84(0.71,1.01)$ \\
\hline Sufficiently active & 559 & $0.52(0.43,0.63)$ & $0.69(0.56,0.86)$ & $0.73(0.61,0.88)$ \\
\hline$p$ value for trend & & $<0.001$ & $<0.001$ & $<0.001$ \\
\hline \multicolumn{5}{|c|}{$\operatorname{CVD}(n=647 ;$ number of cases $=306)$} \\
\hline Inactive & 296 & 1 & 1 & 1 \\
\hline Insufficiently active & 171 & $0.70(0.54,0.93)$ & $0.91(0.67,1.23)$ & $0.90(0.69,1.18)$ \\
\hline Sufficiently active & 180 & $0.61(0.46,0.82)$ & $0.77(0.55,1.07)$ & $0.76(0.58,1.00)$ \\
\hline$p$ value for trend & & $<0.001$ & 0.1 & $<0.05$ \\
\hline \multicolumn{5}{|c|}{ Non-CVD $(n=1,152 ;$ number of cases $=378)$} \\
\hline Inactive & 438 & 1 & 1 & 1 \\
\hline Insufficiently active & 335 & $0.62(0.49,0.79)$ & $0.70(0.54,0.91)$ & $0.81(0.64,1.02)$ \\
\hline Sufficiently active & 379 & $0.48(0.37,0.62)$ & $0.61(0.45,0.82)$ & $0.71(0.56,0.91)$ \\
\hline$p$ value for trend & & $<0.001$ & $<0.001$ & 0.005 \\
\hline
\end{tabular}

SD, standard deviation; CVD, cardiovascular disease; BMI, body mass index; HRs, hazard ratios; CIs, confidence intervals; PA, physical activity. ${ }^{\mathrm{a} C o x}$ proportional hazards regression model adjusting for age, sex, CVD, comorbidity index, smoking status, pack-years, perceived general health, perceived health 1 year before interview, hypertension, activities of daily living score, general health questionnaire score, mini-mental state examination score, BMI, ethnicity, marital status, living alone, employment, education, personal income, and household income. ${ }^{b}$ Weighted Cox proportional hazards regression model based on the propensity score for PA category.

sufficiently active, $171(26.4 \%)$ were insufficiently active, and 296 (45.7\%) were inactive; among the 1,152 nonCVD subjects, the PA distribution was 379 (32.9\%), 335 $(29.1 \%)$, and $438(38.0 \%)$, respectively $(p=0.01)$ (Fig. 1 ).

The mean (25th-75th percentile) follow-up period was 8.8 (7.6-10.8) years. During that period, 684 (38.0\%) participants died. The mean age (standard deviation) of the decedents was 83.4 (6.9) years. Propensity score-adjusted cumulative mortality rates across PA categories and CVD status are presented in Figure 2. In general, non-CVD participants had lower mortality rates than

Physical Activity and Mortality in Older Adults
CVD participants. In addition, PA was associated with lower mortality rates, in a dose-response fashion, in both CVD and non-CVD groups. Notably, the adjusted mortality rate in inactive non-CVD participants was comparable to that of sufficiently active CVD participants, particularly during the later follow-up stage. In relative terms, the unadjusted HRs for mortality in the entire cohort were 0.64 (95\% CI, 0.54-0.77) in insufficiently active and 0.80 (95\% CI, 0.66-0.97) in sufficiently active participants, compared with inactive individuals ( $p$ for trend $<0.001)$. In the propensity score-weighted model, the 
HRs were 0.84 (95\% CI, 0.71-1.01) and 0.73 (95\% CI, $0.61-0.88$ ), respectively ( $p$ for trend $<0.001$ ). Similar results were obtained using a traditional multivariable adjustment (Table 2). Among participants with CVD, the HRs in the propensity score-weighted model were 0.90 (95\% CI, 0.69-1.18) in insufficiently active and 0.76 (95\% CI, 0.58-1.00) in sufficiently active participants, compared with inactive individuals ( $p$ for trend $<0.05$ ); among non-CVD participants, the HRs were 0.81 (95\% CI, 0.641.02 ) and 0.71 (95\% CI, 0.56-0.91), respectively ( $p$ for trend $=0.005)$ (Table 2). The association between PA and mortality did not differ statistically between participants with and without underlying CVD, on neither multiplicative scale $(p$ for interaction $=0.36)$ nor additive scale $(p$ for interaction $=0.58)$.

Applying spline interpolation technique, a nonlinear relationship between the total time of PA (minutes per week) and mortality was detected (Fig. 3). As the total weekly minutes increased, the risk for mortality decreased, with a maximal protective effect observed around 150 PA min per week. Beyond 150 PA min per week, no further improvement in survival was found.

\section{Discussion}

In a nationwide cohort of Israeli individuals with an average age of 75 years, over $40 \%$ were physically inactive and another $30 \%$ were insufficiently active. PA was inversely associated with propensity score-adjusted longterm mortality risk. The association was similar between individuals with and without CVD at study entry. Remarkably, CVD patients who performed sufficient PA had a comparable mortality risk to inactive individuals free of CVD. To our knowledge, this is among the first studies to specifically explore long-term mortality in relation to PA among older adults aged $\geq 65$ years while adequately accounting for a wide range of potential confounders. Furthermore, whether the weekly amount of PA recommended by the ACSM for healthy adults aged 18-65 years should be similarly applied to older agegroups needed clarification. In the present study, we were able to show a survival benefit starting at virtually any amount of weekly PA.

In a multinational panel database that included representative samples of community-based populations from 19 countries in Europe, the overall prevalence of inactivity among individuals aged 55 or older was $12.5 \%$, geographically ranging from $4.9 \%$ (Sweden) to $29 \%$ (Portugal) [24]. Using data from 3 leading national sur-

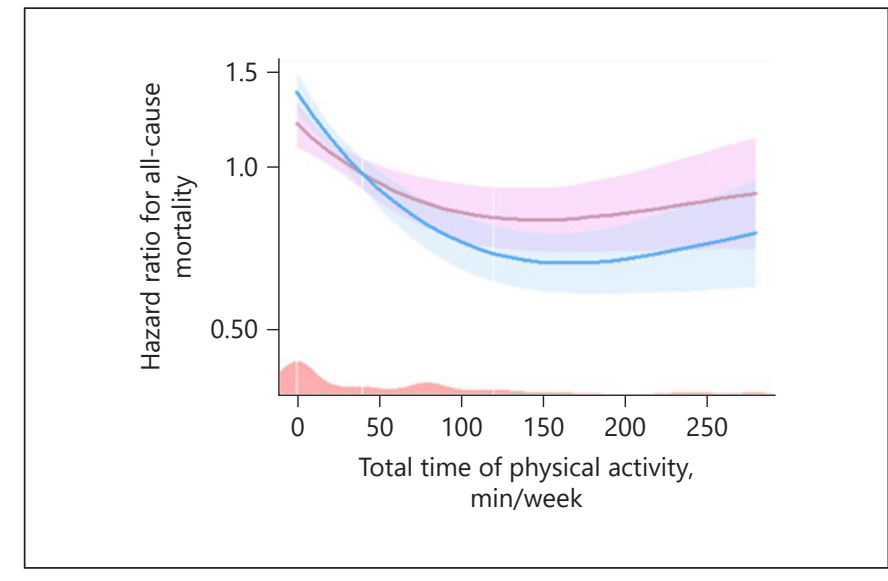

Fig. 3. Spline-based HRs and 95\% CIs for mortality associated with the total time of PA (minutes per week). Spline-based HRs (95\% CIs) for mortality associated with total time of PA (minutes per week) derived from unadjusted (blue) and multivariable-adjusted (purple) Cox models. The latter is adjusted for age, sex, comorbidity index, smoking, perceived general health, perceived health 1 year preceding the baseline interview, hypertension, ADL score, MMSES score, BMI, ethnicity, marital status, employment, education, and household income. The total amount of PA was calculated as the sum of all leisure activities reported at the baseline interview, based on duration and frequency of each activity. The histogram at the bottom shows the distribution of PA total time. The highest $5 \%$ of total time has been trimmed for visualization purposes. HRs, hazard ratios; CIs, confidence intervals; BMI, body mass index; PA, physical activity.

veys in the USA, the estimated prevalence of adults aged 65 and over not meeting the PA guideline was higher but differed by survey, ranging from 36.1 to $60.9 \%$ [25]. In our nationally representative sample of adults aged $\geq 65$ years, $68.9 \%$ did not meet the PA guideline (including $40.8 \%$ who did not engage in any PA). These proportions were even higher for participants with prevalent CVD.

Several previous studies have evaluated the possible health benefits of PA among participants with a variety of different morbidities [26, 27]. To this end, there is a lack of consensus regarding tailored PA guidelines for specific patient populations [27]. In particular, the modifying role of pre-existing CVD in the association between PA and mortality remains unclear, with only a few studies that are directly addressing this question $[13,14]$. Bembom et al. [13] investigated 2,074 persons aged 54 years and older living in and around Sonoma, CA, and showed that the association between PA and mortality was independent of the presence or absence of underlying CVD. However, this study has several limitations, including a short follow-up period of 2-years and relatively "young" 
older adults who were, on average, better educated and more health-conscious relative to the community from which they were drawn. This led to a low mortality rate. From a methodological point of view, the inactive participants were analyzed together with the insufficiently active participants, thereby limiting generalizability. In contrast to the Sonoma study, an analysis utilizing data from the National Health Insurance Services-Health Screening Cohort in Korea recently suggested that individuals with CVD may benefit from PA to a greater extent than healthy subjects without CVD [14]. Again, some methodological issues are important to mention in this context. First, the study had a short median follow-up time of 5.9 years, with relatively young median age of $<60$ years. Also, functional status, mental health, and cognitive performance were not adjusted for. Moreover, CVD participants were, on average, 6 years older than nonCVD participants, calling into question this interaction finding.

A recent meta-analysis showed that among adults aged $\geq 60$ years, even a lower than recommended amount of PA was associated with a $22 \%$ mortality risk reduction, compared with $28 \%$ reduction in those meeting the recommendations [28]. Our results extend this finding by showing a graded relationship between PA and long-term survival in elderly people with and without pre-existing CVD. The possible benefits of PA can be attributed to reverse causation, when inactivity is the result of the presence of advanced disease or disability at the time of the survey $[9,29]$. Studies of older adults are particularly vulnerable to reverse causation [29] because older individuals who are healthy enough to preform PA are more likely to experience a reduced mortality risk irrespective of their PA level, a concept referred to as a "healthy exerciser bias" [9]. This issue pertains also to the question of whether or not the presence of CVD influences the engagement in PA. In order to minimize this bias, we used a propensity score adjustment approach [30]. In this context, the wealth and diversity of social and health-related information available in our dataset constitute a major methodological strength of this study. Specifically, attributes of aging, such as poor self-rated health, functional limitations, cognitive impairment, and decreased psychological functioning [4], may serve as important confounders because they are strongly associated with both PA engagement $[5,8,31]$ and mortality [7]. SES constitutes another important confounder in this context, being related to both exposure [31] and outcome [32]. Nevertheless, many previous investigations conducted among older adults did not address these potential key con-

Physical Activity and Mortality in Older Adults founders in their analyses. For example, a few studies on the association between PA and mortality in the elderly population have incorporated measures of mental health $[33,34]$, self-rated health $[33,35]$, functional limitations, or any additional SES aspects beyond education [14], which may result in substantial residual confounding [36, 37].

Some limitations of our study should be acknowledged. We relied on self-reported data on both PA and CVD that likely resulted in some exposure misclassification. Although objectively measured PA appears to provide slightly more consistent results than self-reported PA [38], the latter was previously found to be reasonably valid, including among aged individuals [39]. Nonetheless, it is advised that future studies shall use both selfreported and objectively measured PA to gain the most complete PA information [38]. Regarding self-reported CVD in our study, cardiovascular events are unlikely to be missed when those are self-reported. Indeed, an Australian study showed a high accuracy of self-reported CVD events in comparison to medical record adjudication and a statewide hospital morbidity database [40]. Exposure data in our study were reported only once, at baseline, and changes in PA levels and/or CVD status over the course of the follow-up period were not captured. This may result in underestimation of the true associations [3]. Generally, PA can be divided into leisure-time PA and nonexercise activity thermogenesis, such as transportation, occupational, and household activities. Only the former was assessed in our study, and we did not address the different modalities of PA performed (e.g., walking, cycling, swimming, etc.). Further research, adequately powered to assess specific PA domains, such as frequency, duration, intensity, and activity type, is therefore warranted.

\section{Conclusions}

In this nationwide survey of older adults, close to half of the participants were inactive and $~ 70 \%$ did not meet the guideline for PA. Our survival analysis supports the importance of performing PA, even below the recommended threshold, by showing an inverse relationship with long-term mortality risk among adults aged 65 years and over. Elderly people, regardless of their CVD status, can benefit from any amount of PA, with a maximum survival advantage supposedly achieved at around 150 min of exercise per week. These findings support the importance of PA as part of healthy aging. 


\section{Statement of Ethics}

All aspects of the study were conducted according to the World Medical Association Declaration of Helsinki and approved by the Institutional Ethics Committees at both Sheba Medical Center (3546-16-SMC) and Tel Aviv University (No. 362); written informed consent was obtained for every participant.

\section{Conflict of Interest Statement}

The authors report no competing interests.

\section{Funding Sources}

This work was supported in part by Grant 3-12787 from the Chief Scientist Office, Israel Ministry of Health (principal investigator, Y.G.), the Stanley Steyer Institute for Cancer Epidemiology and Research (principal investigator, Y.G.), and the Lilian and Marcel Pollak Chair in Biological Anthropology, Sackler Faculty of Medicine, Tel Aviv University, Israel (principal investigator, Y.G.).

\section{References}

1 Lollgen H, Bockenhoff A, Knapp G. Physical activity and all-cause mortality: an updated meta-analysis with different intensity categories. Int J Sports Med. 2009;30(3):213-24.

2 Arem H, Moore SC, Patel A, Hartge P, Berrington de Gonzalez A, Visvanathan $\mathrm{K}$, et al. Leisure time physical activity and mortality: a detailed pooled analysis of the dose-response relationship. JAMA Intern Med. 2015;175(6): 959-67.

3 Gerber Y, Myers V, Goldbourt U, Benyamini Y, Scheinowitz M, Drory Y. Long-term trajectory of leisure time physical activity and survival after first myocardial infarction: a population-based cohort study. Eur J Epidemiol. 2011;26(2):109-16.

4 Meng X, D'Arcy C. Successful aging in Canada: prevalence and predictors from a population-based sample of older adults. Gerontology. 2014;60(1):65-72.

5 Chad KE, Reeder BA, Harrison EL, Ashworth NL, Sheppard SM, Schultz SL, et al. Profile of physical activity levels in community-dwelling older adults. Med Sci Sports Exerc. 2005; 37(10):1774-84.

6 Kelman HR, Thomas C, Kennedy GJ, Cheng J. Cognitive impairment and mortality in older community residents. Am J Public Health. 1994;84(8):1255-60.

7 Maier H, Smith J. Psychological predictors of mortality in old age. J Gerontol B Psychol Sci. 1999;54(1):P44-54.

8 Notthoff N, Reisch P, Gerstorf D. Individual characteristics and physical activity in older adults: a systematic review. Gerontology. 2017;63(5):443-59.

\section{Author Contributions}

O.S., G.C., and Y.G. had full access to all the data in the study and take responsibility for the integrity of the data and the accuracy of the data analysis. Contributions in conception or design of the work provided by O.S., G.C., and Y.G.; contributions in the acquisition, analysis, or interpretation of data for the work given to all authors; statistical analysis performed by O.S., G.C., and Y.G.; drafting of the work done by O.S., G.C., and Y.G.; revising the draft critically for important intellectual content done by all authors; final approval of the version to be published given by all authors.

\section{Data Availability Statement}

Anonymized data and materials have been made publicly available at the Israel Ministry of Health website (https://www.health. gov.il/UnitsOffice/ICDC/mabat/Pages/Mabat_Gold.aspx).
9 Kujala UM. Is physical activity a cause of longevity? It is not as straightforward as some would believe. A critical analysis. Br J Sports Med. 2018;52(14):914-8.

10 Robinson MM, Dasari S, Konopka AR, Johnson ML, Manjunatha S, Esponda RR, et al. Enhanced protein translation underlies improved metabolic and physical adaptations to different exercise training modes in young and old humans. Cell Metab. 2017;25(3):58192.

11 Afilalo J, Alexander KP, Mack MJ, Maurer MS, Green P, Allen LA, et al. Frailty assessment in the cardiovascular care of older adults. J Am Coll Cardiol. 2014;63(8):747-62.

12 Orkaby AR, Forman DE. Physical activity and CVD in older adults: an expert's perspective. Expert Rev Cardiovasc Ther. 2018;16(1):1-10.

13 Bembom O, van der Laan M, Haight T, Tager I. Leisure-time physical activity and all-cause mortality in an elderly cohort. Epidemiology. 2009;20(3):424-30.

14 Jeong SW, Kim SH, Kang SH, Kim HJ, Yoon $\mathrm{CH}$, Youn TJ, et al. Mortality reduction with physical activity in patients with and without cardiovascular disease. Eur Heart J. 2019; 40(43):3547-55.

15 Piercy KL, Troiano RP, Ballard RM, Carlson SA, Fulton JE, Galuska DA, et al. The physical activity guidelines for Americans. JAMA. 2018;320(19):2020-8.

16 Goshen A, Goldbourt U, Shohat T, Shimony T, Keinan-Boker L, Gerber Y. Diet quality in relation to healthy ageing: the Israeli Longitudinal Study on Aging (ILSA)-a study protocol. BMJ Open. 2019;9(4):e024673.
17 Mabat Zahav National Health and Nutrition Survey Age 65 and over 2005-2006. Available from: https: //www.health.gov.il/PublicationsFiles/Mabat_2005-2006-a.pdf.

18 Mabat Zahav Questionnaire English CODED. Available from: https://www.health.gov. il/UnitsOffice/ICDC/mabat/Documents/ Questionnaire_Mabat_Zahav_English_ CODED.pdf.

19 Cohen G, Steinberg DM, Keinan-Boker L, Shaked O, Goshen A, Shimony T, et al. Leisuretime physical activity and cancer risk among older adults: a cohort study. Mayo Clin Proc Innov Qual Outcomes. 2020;4(2):115-25.

20 American College of Sports Medicine Guidelines for Exercise Testing and Prescription. Available from: https://www.acsm.org/readresearch/books/acsms-guidelines-for-exercise-testing-and-prescription.

21 Austin PC. The use of propensity score methods with survival or time-to-event outcomes: reporting measures of effect similar to those used in randomized experiments. Stat Med. 2014;33(7):1242-58.

22 Xu S, Ross C, Raebel MA, Shetterly S, Blanchette C, Smith D. Use of stabilized inverse propensity scores as weights to directly estimate relative risk and its confidence intervals. Value Health. 2010;13(2):273-7.

23 Hernán MARJ. Causal inference: what if. Boca Raton: Chapman \& Hall/CRC; 2020.

24 Gomes M, Figueiredo D, Teixeira L, Poveda V, Paúl C, Santos-Silva A, et al. Physical inactivity among older adults across Europe based on the SHARE database. Age Ageing. 2016; 46(1):71-7. 
25 Keadle SK, McKinnon R, Graubard BI, Troiano RP. Prevalence and trends in physical activity among older adults in the United States: a comparison across three national surveys. Prev Med. 2016;89:37-43.

26 Rock CL, Doyle C, Demark-Wahnefried W, Meyerhardt J, Courneya KS, Schwartz AL, et al. Nutrition and physical activity guidelines for cancer survivors. CA Cancer J Clin. 2012; 62(4):243-74.

27 Liu Y, Shu XO, Wen W, Saito E, Rahman MS, Tsugane S, et al. Association of leisure-time physical activity with total and cause-specific mortality: a pooled analysis of nearly a half million adults in the Asia Cohort Consortium. Int J Epidemiol. 2018;47(3):771-9.

28 Hupin D, Roche F, Gremeaux V, Chatard JC, Oriol M, Gaspoz JM, et al. Even a low-dose of moderate-to-vigorous physical activity reduces mortality by $22 \%$ in adults aged $>/=60$ years: a systematic review and meta-analysis. Br J Sports Med. 2015;49(19):1262-7.

29 Wade KH, Richmond RC, Davey Smith G. Physical activity and longevity: how to move closer to causal inference. Br J Sports Med. 2018;52(14):890-1.
30 Lunceford JK. Stratification and weighting via the propensity score in estimation of causal treatment effects: a comparative study. Stat Med. 2017;36(14):2320.

31 Picorelli AM, Pereira LS, Pereira DS, Felício $\mathrm{D}$, Sherrington C. Adherence to exercise programs for older people is influenced by program characteristics and personal factors: a systematic review. J Physiother. 2014;60(3): 151-6.

32 Lantz PM, Golberstein E, House JS, Morenoff J. Socioeconomic and behavioral risk factors for mortality in a national 19-year prospective study of U.S. adults. Soc Sci Med. 2010;70(10): 1558-66.

33 Ueshima K, Ishikawa-Takata K, Yorifuji T, Suzuki E, Kashima S, Takao S, et al. Physical activity and mortality risk in the Japanese elderly: a cohort study. Am J Prev Med. 2010 38(4):410-8.

$34 \mathrm{Wu}$ CY, Hu HY, Chou YC, Huang N, Chou YJ, Li CP. The association of physical activity with all-cause, cardiovascular, and cancer mortalities among older adults. Prev Med. 2015;72:23-9.
35 Mok A, Khaw KT, Luben R, Wareham N, Brage S. Physical activity trajectories and mortality: population based cohort study. BMJ. 2019;365:12323.

36 Braveman PA, Cubbin C, Egerter S, Chideya S, Marchi KS, Metzler M, et al. Socioeconomic status in health research: one size does not fit all. JAMA. 2005;294(22):2879-88.

37 Myers V, Drory Y, Goldbourt U, Gerber Y. Multilevel socioeconomic status and incidence of frailty post myocardial infarction. Int J Cardiol. 2014;170(3):338-43.

38 Skender S, Ose J, Chang-Claude J, Paskow M, Brühmann B, Siegel EM, et al. Accelerometry and physical activity questionnaires: a systematic review. BMC Public Health. 2016;16: 515.

39 Orsini N, Bellocco R, Bottai M, Hagströmer M, Sjöström M, Pagano M, et al. Validity of self-reported total physical activity questionnaire among older women. Eur J Epidemiol. 2008;23(10):661-7.

40 Barr EL, Tonkin AM, Welborn TA, Shaw JE. Validity of self-reported cardiovascular disease events in comparison to medical record adjudication and a statewide hospital morbidity database: the AusDiab study. Intern Med J. 2009;39(1):49-53. 